\title{
Time Development of Voltage Frequency Dependence of Partial Discharge Activity in Voids
}

\author{
T. G. Aakre ${ }^{1}$, E. Ildstad ${ }^{1} \&$ S. Hvidsten ${ }^{2}$ \\ ${ }^{I}$ NTNU/ Department of Electrical Power Engineering, Trondheim, Norway \\ ${ }^{2}$ SINTEF Energy Research, Trondheim, Norway
}

\begin{abstract}
Condition assessment of high voltage equipment based on partial discharge measurements is often performed after a voltage pre-conditioning period. The aim of this paper is to present results from experimental examinations of time variance of partial discharge activity and to propose physical explanations of the phenomena observed. Experiments were performed on laboratory made $3 \mathrm{~mm}$ thick discs of generator bar insulation, consisting of mica and glass fiber reinforced epoxy with a $0.5 \mathrm{~mm}$ thick cylindrical void surfaces of $10 \mathrm{~mm}$ in diameter. The effect of conducting and insulating void surfaces was examined using copper tape as upper and lower electrodes of the voids. All objects were tested by 12 one-minute long AC voltage frequency sweeps at frequencies from $50 \mathrm{~Hz}$ to $0.1 \mathrm{~Hz}$ distributed in time from start of the experiment, after initial onehour constant $50 \mathrm{~Hz}$ voltage application and during object shortcircuiting for $20 \mathrm{~h}$. The main result shows that in case of insulating voids the apparent charges vanished after the onehour constant $50 \mathrm{~Hz}$ voltage application. After a grounding period of 5 minutes, the charge magnitudes slowly increased with time until reaching steady state after about 4-8 hours. Test objects with conductive void surfaces showed such reduction in case of PD testing at $0.1 \mathrm{~Hz}$ only. At voltage frequencies above $10 \mathrm{~Hz}$ the measured PD magnitudes were found to be nearly constant, close to the expected high theoretical value. This indicate that PD by-products strongly affected the void resistivity and thereby affect the PD activity of insulating voids. These by-products are temporary and disappear with time.
\end{abstract}

\section{Introduction}

To avoid temporary partial discharge (PD) events and to ensure stable void conditions during diagnostic PD-testing, IEC 60034-27 [1] and IEEE std 1434 [2] suggest a procedure for preconditioning of stator bar insulation. It is recommended to use at least 1-15 minutes of pre-conditioning at maximum test voltage. Recommended duration is, however, flexible, as it is stated that pre-conditioning up to 1 hour may be preferable. For example it is reported that in case of polycarbonate insulated samples stable PD conditions were reached after 1.5 hours of pre-conditioning [3].

The main purpose of this work is to experimentally investigate how PD parameters change with varying frequency AC test voltage after different durations of short-circuiting time after the one-hour pre-conditioning period. The observed phenomena are discussed with respect to a classical theory of interpretation of PD measurements.

\section{Theoretical model}

Equations applied in this chapter are based on the PD characteristics of new samples with no preceding PD activity. Examples on how preceding PD activity might change the void properties are shown in Chapter 2.3.

PD arcs in a void are assumed to last for a very short time, most probably a few nanoseconds [4], i.e. an applied test voltage of $50 \mathrm{~Hz}$ can be considered constant during the PD arc. This means that any voltage frequency dependence of PD activity must be caused by changes occurring before the first PD occurrence.

\subsection{Frequency dependent voltage across the void}

A void encapsulated in a partly conducting dielectric can be modelled by a modified abc-model, as shown in Figure 1. Here the capacitors $C_{\mathrm{a}}$ and $C_{\mathrm{b}}$ of the insulation and the void capacitance $C_{\mathrm{c}}$ are considered to have a resistor in parallel.

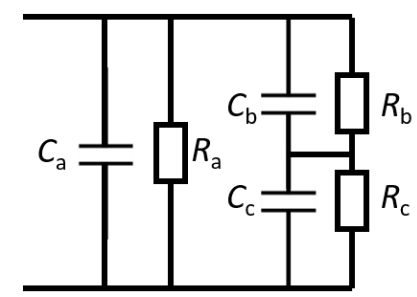

Figure 1: The modified abc-equivalent circuit for interpretation PD activity in a void enclosed in an insulation system with low conductivity.

The voltage across the void $U_{\mathrm{c}}$ is determined by the applied voltage $U_{\mathrm{a}}$

$$
U_{\mathrm{c}}=\frac{Z_{\mathrm{c}}}{Z_{\mathrm{c}}+Z_{\mathrm{b}}} \cdot U_{\mathrm{a}}
$$

where $Z_{\mathrm{b}}$ and $Z_{\mathrm{c}}$ represent the frequency dependent impedance of the involved sections. This equation has a cut-off frequency given by the Maxwell-Wagner relation

$$
f_{\text {cut }- \text { off }} \approx \frac{1}{R C}=\frac{\frac{1}{R_{\mathrm{b}}}+\frac{1}{R_{\mathrm{c}}}}{C_{\mathrm{b}}+C_{\mathrm{c}}}=\frac{R_{\mathrm{b}}+R_{\mathrm{c}}}{R_{\mathrm{b}} R_{\mathrm{c}}\left(C_{\mathrm{b}}+C_{\mathrm{c}}\right)}
$$

A PD can occur when the applied voltage exceeds the breakdown strength of the gas in the void. It is here assumed that the electric field inside the void is homogeneous and that the minimum breakdown voltage $U_{\mathrm{s} 0}$ is given by Paschen law [5]

$$
U_{\mathrm{s} 0}=6.72 \sqrt{p d}+24.36(p d)[\mathrm{kV}]
$$


where $p$ is the pressure and $d$ the gap distance. This expression is valid for $p d$ values in the range from $10^{-2}$ to $5 \cdot 10^{2}$ (bar $\mathrm{cm}$ ).

\subsection{Derived partial discharge parameters}

When the electric field locally is above a threshold value, an electron is needed to start PD initiation (statistic time lag) before the formation of the PD event takes place (formative time lag). The apparent charge $q_{\mathrm{a} 0}$ is measured in the external circuit, and can be modelled by the abc-equivalent, due to the short duration of the PD pulse. In case the entire void is considered discharged

$$
q_{\mathrm{a} 0}=C_{\mathrm{b}} \cdot\left(U_{\mathrm{s}}-U_{\mathrm{r}}\right)
$$

where $C_{\mathrm{b}}$ is the capacitance of the insulation in series with the void, $U_{\mathrm{r}}$ is the remanent voltage after a $\mathrm{PD}$ has occurred and $U_{\mathrm{s}}$ the actual breakdown voltage of the void. There might be a voltage higher than that of equation (3) if no start electron is available when the voltage increased above the threshold limit. A frequency independent time delay $\Delta t$ causes a larger overvoltage at high frequency due to a larger voltage change during the same period compared to low frequency

$$
U_{\mathrm{s}}=U_{0} \cdot \sin \left(\omega\left(t_{\mathrm{PD} 0}+\Delta t\right)\right)
$$

where $U_{0}$ is the maximum void voltage, $t_{\mathrm{PD} 0}=\frac{1}{\omega} \cdot \operatorname{asin}\left(\frac{U_{\mathrm{s} 0}}{U_{0}}\right)$ is found by using $\Delta t=0$ and Paschen voltage.

The PD pulse repetition rate $n$ per half period is rounded down to the nearest digit and given by

$$
n=\left\lfloor 2 \cdot \frac{U_{0}-U_{\mathrm{r}}}{U_{\mathrm{s}}-U_{\mathrm{r}}}\right\rfloor
$$

where $U_{0}$ is the maximum voltage applied across the void during a voltage cycle.

A new alternative approach, suggested here, is to define a socalled apparent repetition rate, $\hat{n}$. This is defined as the sum of all measured apparent charge magnitudes divided by the theoretical apparent charge value

$$
\hat{n}=\frac{1}{q_{\mathrm{a} 0}} \sum q_{\mathrm{a}}
$$

The total measured apparent charge is a parameter that is easy to measure, less influenced by statistical variation than the apparent charge and is directly dependent on the capacitance $C_{\mathrm{b}}$ in series with the void, the maximum void voltage $U_{0}$ and the remanent voltage $U_{\mathrm{r}}$. The total apparent charge is expressed by combining equations (4), (6) and (7):

$$
\sum q_{\mathrm{a}}=\hat{n} \cdot q_{\mathrm{a} 0}=4 \cdot g \cdot C_{\mathrm{b}} \cdot\left(U_{0}-U_{\mathrm{r}}\right)
$$

where $g$ is an area correction factor introduced as the 'relative PD-active' void area.

\subsection{Influence of surface change due to PD activity}

Free electrons, or start electrons, in the void can originate from different sources, such as cosmic radiation, field emission from the void walls with charges in shallow traps [6]. The PD arc reacts with the air in the void and the void surface and can produce various acids, which have been found to highly increase the polymeric void surface conductivity [7, 8]. Such acids are known to self-decompose into neutral components, nitric acid in particular [9]. The increased surface conductivity due to PD arcs is therefore expected to decrease with time after short-circuiting. An increasing surface conductivity on epoxy surfaces caused by PD activity has been shown to change the discharge type from spark to continuous glow discharges [10]. Glow discharges produce a continuous current and can only be measured with photodetectors or pico-amperemeters. A higher surface conductivity reduces the void resistance and hence the void voltage at low frequency. This is demonstrated by the graphs presented in Figure 2, where a sketch of the normalized void voltage based on equation (1) as function of frequency are given for different void relaxation times $\left(\tau_{\mathrm{c}}=R_{\mathrm{c}} C_{\mathrm{c}}\right)$.

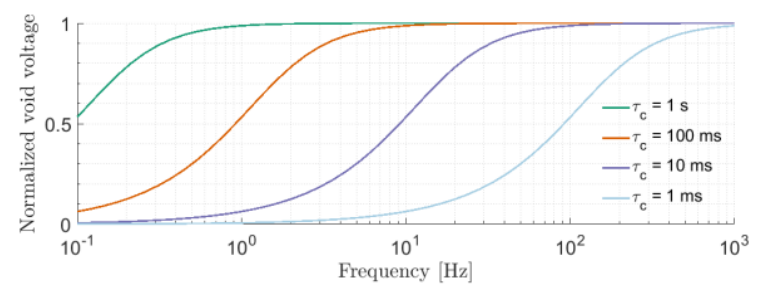

Figure 2: Sketch of relative void voltage for different void relaxation times $\tau_{\mathrm{c}}=R_{\mathrm{c}} C_{\mathrm{c}}$, based on equation (1). The relaxation time of the insulation is chosen high; 1000 s, i.e. representing a low insulation conductivity.

\section{Examined test objects}

The test samples were produced using a commercially available mica tape, which was cured at $160^{\circ} \mathrm{C}$ for $1 \mathrm{~h}$ under a pressure of 3.3 bar into $1.5 \mathrm{~mm}$ thickness. A $10 \mathrm{~mm}$ hole diameter with height of $0.5 \mathrm{~mm}$ was constructed with a metal spacer in the lower part during the curing. The test object types are sketched in Figure 3. The samples had dimensions $100 \times 100 \times 3 \mathrm{~mm}^{3}$. The upper and lower surfaces (indicated with a red line) were either kept insulating, or covered with copper tape of thickness $0.1 \mathrm{~mm}$ giving a reduced void height of $0.3 \mathrm{~mm}$. Three similar samples were made for each type of test object.

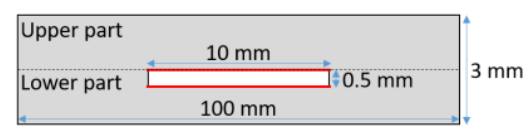

Figure 3: Sketch of test objects. The void surface (red) can be covered with $0.1 \mathrm{~mm}$ thick copper tape.

The samples were clamped between two brass electrodes enclosed in an epoxy cylinder to prevent spurious discharges in the air at the triple point between insulation surface, electrode and air. Additionally, a thin layer of silicone grease was applied between the electrodes and the sample to prevent unintended PD in small voids at the interface between the test object and the metal electrodes. 


\section{Material characterization}

\subsection{Methods}

The complex permittivity of the insulation as function of frequency was determined by dielectric spectroscopy measurements at $200 \mathrm{~V}$ in the frequency range of $1 \mathrm{mHz}$ to $0.1 \mathrm{kHz}$ with IDAX 206 hardware and software.

One test object was modified by connecting thin copper wires to the copper tapes positioned at the surface of the voids. The wire ends were available on the outside of the test objects. The void resistance of this object before and after $1 \mathrm{~h}$ of $10 \mathrm{kV}_{\mathrm{rms}}$ at $50 \mathrm{~Hz}$ was measured using a DC insulation tester at $1 \mathrm{kV}$.

\subsection{Results from material characterization}

The results from material characterization are shown in Table 1 . The DC conductivity was calculated from the complex permittivity at low frequency.

Table 1. Equivalent circuit parameters for a void with diameter $10 \mathrm{~mm}$ and height $0.5 \mathrm{~mm}(0.3 \mathrm{~mm}$ for conducting samples) and insulation thickness of $2.5 \mathrm{~mm}$ based on measured complex permittivity.

\begin{tabular}{ccl}
\hline Parameter & Value & Comment \\
\hline$C_{\mathrm{b}}[\mathrm{pF}]$ & 1.1 & Based on measured permittivity at $1 \mathrm{kHz}$ \\
$C_{\mathrm{c}}[\mathrm{pF}]$ & 1.4 & Based on void geometry $\mathrm{h}=0.5 \mathrm{~mm}$ \\
$C_{\mathrm{c}}[\mathrm{pF}]$ & 2.3 & Based on void geometry $\mathrm{h}=0.3 \mathrm{~mm}$ \\
$R_{\mathrm{b}}[\mathrm{P} \Omega]$ & 5.6 & Based on measured imaginary part of \\
& $>3$ & Measured before 1 hour at $10 \mathrm{kV}$ rms \\
$R_{\mathrm{c}}[\mathrm{T} \Omega]$ & 1 & Measured right after 1 hour at $10 \mathrm{kV}$ \\
$R_{\mathrm{c}}[\mathrm{T} \Omega]$ & $>3$ & Measured after 20 h of object grounding \\
$R_{\mathrm{c}}[\mathrm{T} \Omega]$ & 0.44 & $C_{\mathrm{b}} /\left(C_{\mathrm{b}}+C_{\mathrm{c}}\right)$, insulating samples \\
Voltage divider & 0.32 & $C_{\mathrm{b}} /\left(C_{\mathrm{b}}+C_{\mathrm{c}}\right)$, conducting samples \\
Voltage divider & 0
\end{tabular}

\section{Partial discharge test methods}

\subsection{Experimental setup}

The experimental setup was fully automated and controlled by a LabView program as shown in Figure 4. The hardware is based on a standard PD detection circuit [11]. The input voltage signal from the computer was amplified by a high voltage amplifier, TREK 20/20B, and filtered by a low pass filter $(5 \mathrm{kHz})$ to reduce inherit amplifier noise. The test object $(\sim 20 \mathrm{pF})$ was in parallel with a coupling capacitance $(100 \mathrm{pF})$.

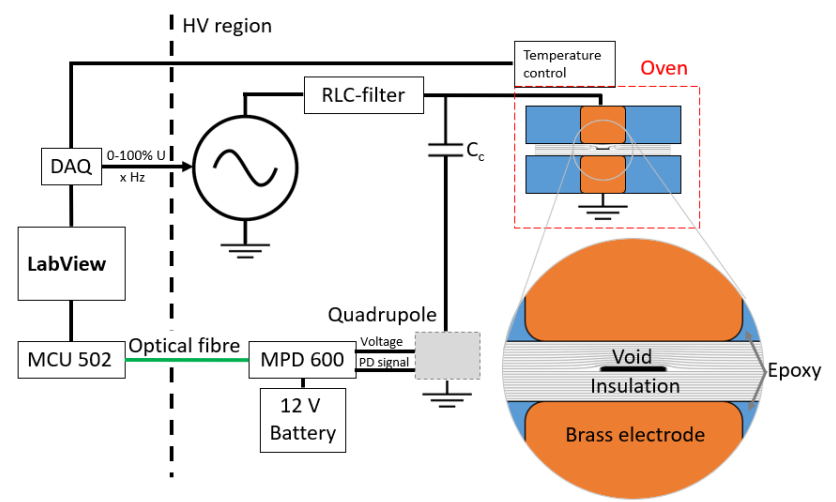

Figure 4: Setup of the PD detection circuit. Everything was controlled by a LabView program.
PD pulses was detected by using a quadrupole in series with the coupling capacitance and processed by the automated PD detection system from Omicron, MPD600 and MCU 502.

The circuit was calibrated with a calibrator that injected a charge corresponding to $100 \mathrm{pC}$ across the test object.

\subsection{Test procedure}

The voltage application on the test samples is sketched in Figure 5. A constant voltage of $10 \mathrm{kV}_{\mathrm{rms}}$ at $50 \mathrm{~Hz}$ was applied the first test hour with a high PD number before short-circuiting for $20 \mathrm{~h}$. PD measurements during voltage frequency sweeps were performed at different time intervals during the test as indicated in the figure. The frequency sweeps consisted of 5 periods at $10 \mathrm{kV}_{\mathrm{rms}}$ at each of the following frequencies: 50,10 , 1, 0.1, 1, 10 and $50 \mathrm{~Hz}$. This included only 35 voltage periods, equivalent to less than a second of $50 \mathrm{~Hz}$ voltage application. It was therefore assumed that the conditions within the void was unaffected by the short period of PD measurements during the frequency sweep. This was controlled by the first and last five $50 \mathrm{~Hz}$ periods in the frequency sweep.

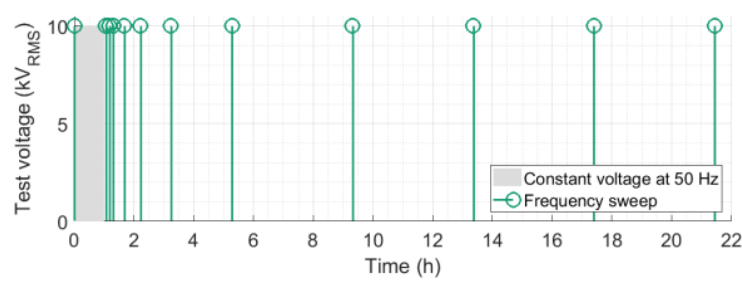

Figure 5: Sketch showing the voltage application versus time. The test object was short-circuited between the frequency sweeps, except between the first and second frequency sweep.

One sample were tested with the frequency sweep order changed, starting at low frequency, to investigate if the test procedure influenced the results.

\section{Results from measurements of time development of PD parameters}

The PD frequency sweep gave the same results regardless of the frequency sequence $(50 \mathrm{~Hz}$ to $0.1 \mathrm{~Hz}$ or $0.1 \mathrm{~Hz}$ to $50 \mathrm{~Hz}$ ) or the position in the frequency sweep (first vs last). Hence, the frequency sweep does not contribute to any measured frequency dependence on the PD characteristics

The measured PDs occurred at different phase locations and with different magnitude. The distribution of PD magnitude at three time instances for the two object types are given as a typical example in Figure 6. The PRPDAs are not shown, but they are symmetric and shifted to the voltage rise, which is typical for void discharges. 


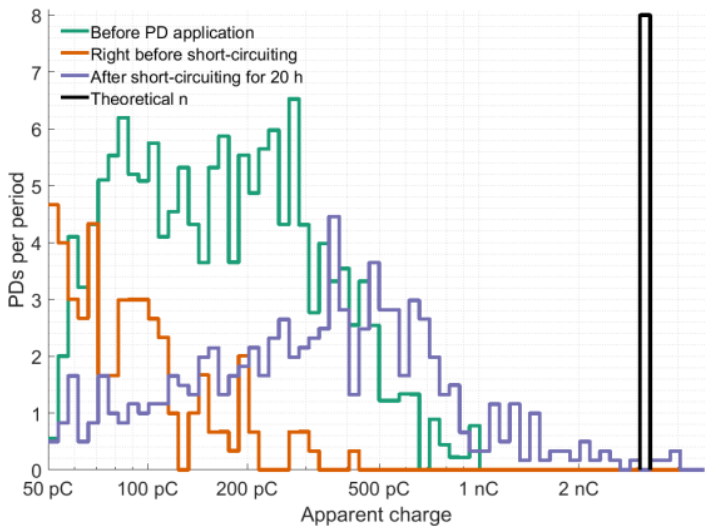

a) Void with insulating surfaces.

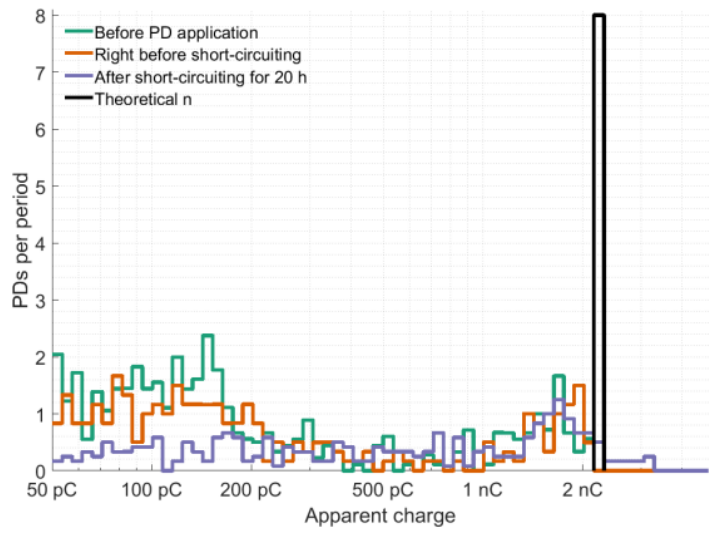

a) Void with copper tape

Figure 6: Histogram of number of PDs per period for both sample types at $50 \mathrm{~Hz}$ based on the average of three samples. It is a similar distribution at all tested frequencies, but with the maximal magnitude slightly different. The theoretical value of 8 per period is based on twice the value obtained in equation (6) for one half period.

The apparent PD repetition rate $\hat{n}$ versus time for different frequency and object type is given in Figure 7. It is a large spread in apparent charge magnitude with many small values and the resulting repetition rate $n$ is very high and thereby useless.

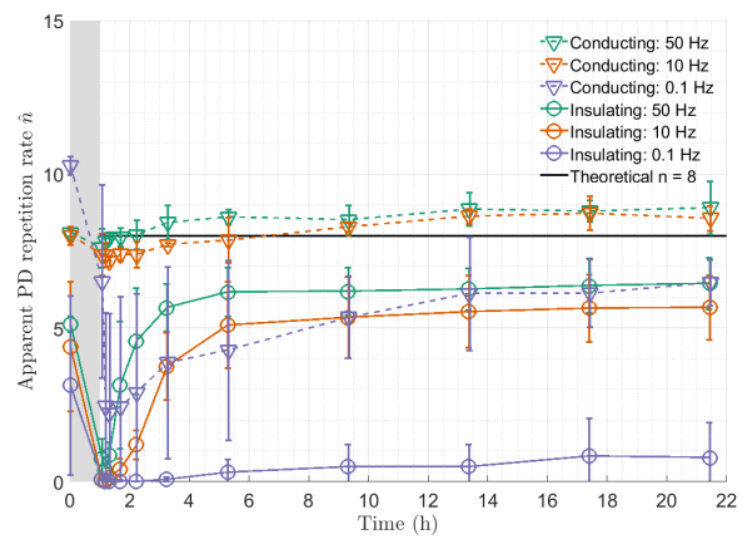

Figure 7: Apparent PD repetition rate $\hat{n}=\sum q_{\mathrm{a}} / q_{\mathrm{a} 0}$ for three parallels (giving the error bars) versus test time. A constant voltage was applied (grey region), with high PDs, for the first test hour. The theoretical value of 8 per period is based on twice the value obtained in equation (6) for one half period.
The normalized maximum detected apparent charge during five voltage periods as function of time for different frequencies and object type is presented in Figure 8. The theoretical value was used as normalization factor, given by equation (4), which is $3.1 \mathrm{nC}$ for insulating objects and $2.2 \mathrm{nC}$ for conductive objects. This difference is due to a reduced void height caused by the thickness of the copper tape.

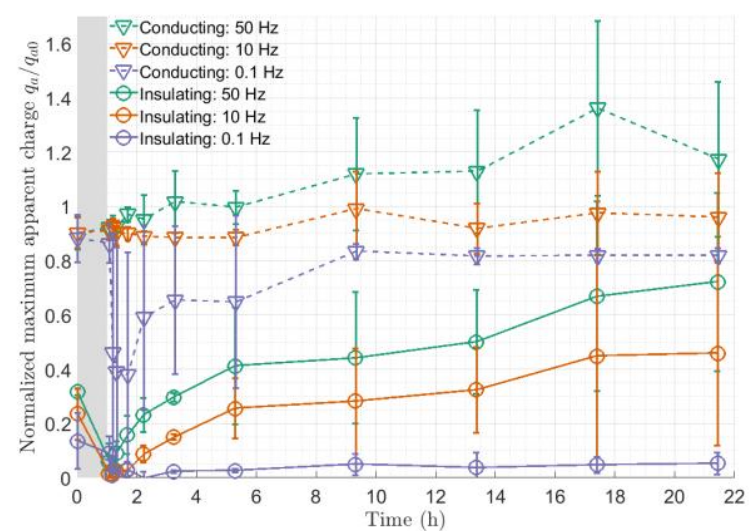

Figure 8: Average normalized maximum apparent charge for three parallels (giving the error bars) versus test time. A constant voltage was applied (grey region), with high PDs, for the first test hour.

The calculated void voltage based on equation (8) is presented in Figure 9 for insulating objects and in Figure 10 for the objects with copper tape. It is a significant influence from the pre-conditioning.

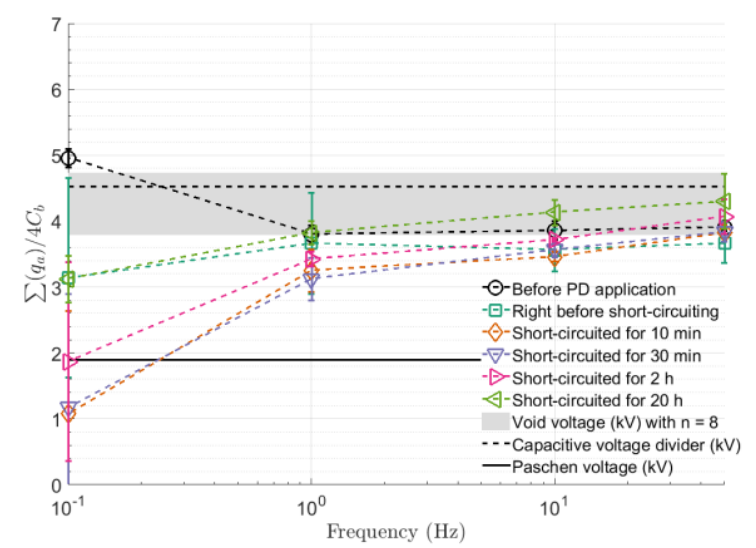

Figure 9: Void voltage as function of frequency at different time instants for samples with copper tape. The y-axis is equal to $g\left(U_{0}-U_{r}\right)$ as defined in equation (8). The void voltage with $\mathrm{n}=8$ per period is based on the voltage region in equation (6) that gives $n=8$. 


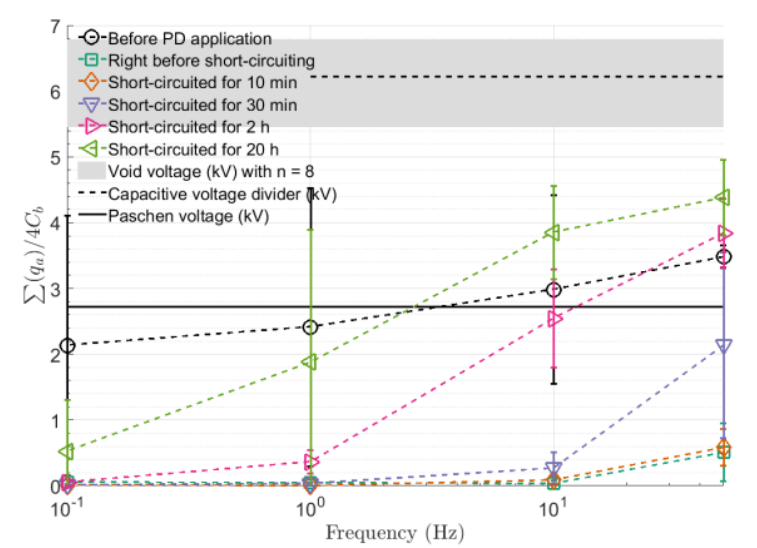

Figure 10: Void voltage as function of frequency at different time instants for samples with insulating surfaces. The y-axis is equal to $g\left(U_{0}-U_{r}\right)$ as defined in equation (8). The void voltage with $\mathrm{n}=8$ per period is based on the voltage region in equation (6) that gives $\mathrm{n}=8$.

\section{Discussion}

The cutoff frequency based on material parameters for new samples, given in Table 1, and equation (2) is less than $1 \mathrm{mHz}$. This means that no frequency dependence is expected. The onehour pre-conditioning lowered the void resistivity, increasing the cutoff frequency to $2 \mathrm{~Hz}$. The pre-conditioning change the void resistivity and is thereby expected to change the voltage frequency response of the void.

Figure 6 shows great difference in distribution of PD magnitude of both object types vs expected theoretical value. A minor spread was expected according to equation (5) with a time lag. The large observed spread cannot be explained by a time lag and it makes no meaning to use the PD repetition rate $n$ to describe the PD activity. The suggested apparent repetition rate $\hat{n}$ is therefore an adequate estimator for how many times the total void area discharges during a voltage period. The small PD magnitudes can be related to edge effects at the edge of the copper tape. Another observation is that the voids with insulating surfaces in a) are more influenced by the constant $50 \mathrm{~Hz}$ voltage application than the voids with copper tape in b).

The calculated apparent repetition rate $\hat{n}$ based on the total apparent charge is presented in Figure 7 for the two object types at different voltage frequencies versus time. The theoretical repetition rate $n$ were expected to be 8 in both objects despite the geometrical difference. The apparent repetition rate for the voids with copper tape was close to the theoretical repetition rate, which indicates that the complete void was discharged as expected. However, the steady-state repetition rate at $0.1 \mathrm{~Hz}$ is only 6 , probable related to a lower void voltage. The apparent repetition rate in case of voids with insulating surfaces was lower than for the samples with copper tape. This can be caused either by a lower void voltage, or a smaller relative 'PD-active area'. The sum of apparent charge divided by four divided by the series capacitance $C_{\mathrm{b}}$, equal to $g\left(U_{0}-U_{r}\right)$ by equation (8), for the objects with copper tape is presented in Figure 9 and with insulating surface in Figure 10. Void voltages below Paschen voltage cannot give PDs and only a fraction of the total area can be 'PD-active'. By assuming a pure capacitive divider, the area correction factor $g$ for the insulating object is: $0.70,0.62,0.30$ and 0.08 for $50,10,1$ and $0.1 \mathrm{~Hz}$ respectively. This corresponds to a reduced 'PD-active' void diameter of 8.4, 7.9, 5.5 and $2.9 \mathrm{~mm}$. The reduction in 'PD-active' area provides still a too high apparent charge if the complete reduced area discharges during a PD, compared to the measured maximum apparent charge at these frequencies shown in Figure 8. This strongly indicates that the voids with insulating surfaces have a smaller frequency dependent PD-active discharge area.

The pre-conditioning lowered the void resistance, as also measured by direct methods reported in Table 1 . The resistance reduction was only temporary as it restored to original state after a long short-circuited period. The reduced void voltage enables less discharges to be produced, as is shown in Figure 7 and a smaller maximum apparent charge as is shown in Figure 8. This effect was largest for the voids with insulating surfaces, which indicates that the properties of the insulating surfaces were changed by the PD activity during constant $50 \mathrm{~Hz}$ voltage application. It was a relatively large frequency dependence, in which the void was almost short-circuited at low frequency. The frequency dependent PD activity at different time instants in Figure 10 for the voids with insulating surfaces can be described by a varying relaxation time. By comparing it with Figure 2 it is seen that the overall trend was that the relaxation time started high, reduced to a low value after the pre-conditioning and then gradually recover with time. This indicates, by a lower PD activity, that the void resistivity decreased with pre-conditioning and increased when grounded. One possible explanation is the presence of PD by-products such as acids, as seen in [7, 8], and following gradual self-decomposition of the acids when the test object was short-circuited. The effect of pre-conditioning was only important for voids with insulating surfaces as there were a large time and frequency dependence in Figure 10 and a low frequency dependence in Figure 9.

Hudon et al. [10] studied PD activity during a long constant AC voltage application with PD and described a transition from spark to glow discharges after a long time. This was correlated to an increased surface conductivity caused by PD activity. The increased surface conductivity in voids, in contrast to [10] with no void walls, short-circuit the void and it is possible that glow discharges are not present in voids. However, a transition to glow discharges can explain a reduced void discharging area and can be interesting to investigate in further works. This study had not the equipment to follow this track any further.

\section{Conclusions}

- The measured repetition rate was much higher than the theoretical value $n$. A more convenient parameter was the estimator apparent repetition rate, $\hat{n}$, which was introduced here. It sums up all the apparent charge during a voltage cycle, normalized to the theoretical apparent charge, and was close to the theoretical value for the repetition rate.

- The pre-conditioning period with PD activity changed the void such that the PDs extinguished at low frequency. This can be explained by a reduced void resistance due to byproducts from PD activity.

- The void change due to pre-conditioning was only temporary as the PD activity slowly restored to the original state when short-circuited. This can be explained by gradual 
neutralization of the by-products from PD process that initially reduced the void voltage.

\section{Acknowledgement}

This work is founded by the project "Hydrogenerator Stator Winding Insulation Assessment". The project is supported by The Research Council of Norway (Project No. 255099/E20), and industrial partners.

\section{References}

[1] IEC TS 60034-27:2006, Off-line partial discharge measurements on the stator winding insulation of rotating electrical machines, 2006.

[2] IEEE Std 1434-2014 (Revision of IEEE Std 1434-2000), IEEE Guide for the Measurement of Partial Discharges in AC Electric Machinery, 2014.

[3] C. Forssen and H. Edin, "Partial discharges in a cavity at variable applied frequency part 1: measurements," IEEE Transactions on Dielectrics and Electrical Insulation, vol. 15, no. 6, pp. 1601-1609, 2008.

[4] R. Morrow and J. J. Lowke, "Streamer propagation in air," Journal of Physics D: Applied Physics, vol. 30, no. 4, p. 614, 1997.

[5] E. Kuffel and W. S. Zaengl, High-voltage engineering: fundamentals. Pergamon Press, 1984.

[6] L. Niemeyer, "A Generalized Approach to Partial Discharge Modeling," IEEE Transactions on Dielectrics and Electrical Insulation, Article vol. 2, no. 4, pp. 510-528, 1995.

[7] C. Hudon, R. Bartnikas, and M. R. Wertheimer, "Surface conductivity of epoxy specimens subjected to partial discharges," in Conference Record of IEEE International Symposium on Electrical Insulation, pp. 153-155, 1990.

[8] C. Hudon, R. Bartnikas, and M. R. Wertheimer, "Analysis of degradation products on epoxy surfaces subjected to pulse and glow type discharges," in 1991 Annual Report. Conference on Electrical Insulation and Dielectric Phenomena, 1991, pp. 237-243.

[9] G. D. Robertson, D. M. Mason, and W. H. Corcoran, "The Kinetics of the Thermal Decomposition of Nitric Acid in the Liquid Phase," The Journal of Physical Chemistry, vol. 59, no. 8, pp. 683-690, 1955/08/01 1955.

[10]C. Hudon, R. Bartnikas, and M. R. Wertheimer, "Spark-to-glow Discharge Transition due to Increased Surface Conductivity on Epoxy Resin Specimens," IEEE Transactions on Electrical Insulation, Article vol. 28, no. 1 , pp. 1-8, 1993.

[11]IEC 60270:2000, High-voltage test techniques - Partial discharge measurements 2000. 